04

\title{
О предельном токе разряда электродугового плазмотрона постоянного тока с трубчатыми электродами
}

\author{
(c) В.М. Кулыгин, А.В. Переславцев, С.С. Тресвятский \\ Национальный исследовательский центр „Курчатовский институт“, \\ 123182 Москва, Россия \\ e-mail: Kulygin_VM@nrcki.ru
}

(Поступило в Редакцию 24 июня 2017 г.)

\begin{abstract}
Имеющиеся в литературе сведения о наличии предельного тока на катодную вставку плазмотрона и связи его величины с диаметром этой вставки обсуждены на основе предположения о том, что это соответствие связано с достижением критической температуры поверхности вставки, при которой происходит резкое увеличение скорости испарения материала.
\end{abstract}

DOI: $10.21883 /$ JTF.2018.03.45591.2404

\section{Введение}

Экспериментальные данные, получаемые при работе с плазмотронами, обычно носят интегральный характер, поэтому суждения о деталях механизма рабочего процесса можно делать лишь косвенно, привлекая модельные физические соображения и сопоставляя полученные результаты с результатами измерений. В настоящей работе мы воспользуемся таким подходом для анализа прикатодных процессов в электродуговом плазмотроне постоянного тока.

В работе [1] устанавливается соответствие между диаметром используемой катодной вставки и предельным током дуги плазмотрона. В табл. 1 приведены соответствующие цифровые данные. Превышение указанных предельных значений ведет к резкому уменьшению срока службы катодной вставки

Имея в виду этот результат, обсудим возможный механизм, обусловливающий существование такого порога. Ранее [2] было установлено, что скорость испарения гафниевой вставки, определяющая ресурс ее работы, существенно меньше скорости испарения чистого гафния в вакуум. Было сделано предположение о возможности влияния на замедление процесса испарения материала вставки двух факторов:

- наличия вблизи ее поверхности давления рабочего газа,

- наличия на этой поверхности пленки оксида и нитрида гафния.

Пороговый характер обсуждаемого явления позволяет предположить, что упомянутый предел определяется достижением некоторого критического значения температуры поверхности, при котором происходит резкое увеличение скорости испарения материала катодной

Таблица 1.

\begin{tabular}{c|c|c|c|c}
\hline Диаметр вставки, cm & 0.16 & 0.2 & 0.25 & 0.30 \\
\hline Предельный ток дуги, А & 80 & 200 & 300 & 400
\end{tabular}

вставки. Логично поэтому принять в качестве рабочей гипотезы определяющее действие второго из названных факторов ограничения испарения: наличием оксиднонитридной корки на поверхности расплавленного слоя материала, а критической температурой счесть температуру плавления этой корки (наиболее тугоплавкой нитридной, $3063 \mathrm{~K}$ ).

\section{Рабочая модель и результаты расчета}

Прикатодную область дуги плазмотрона можно условно разделить на три части.

- Поверхность катодной вставки, нагрев которой производится потоком падающих (и рекомбинирующих) ионов и излучением из дуговой плазмы, а отвод тепла в основном эмиссией электронов и теплопроводностью, так как испарение материала и тепловое излучение дают незначительный вклад. (В азоте воздуха при атмосферном давлении потери на излучение составляют одиннесколько процентов от вкладываемой мощности.)

- Прикатодный слой, примыкающий непосредственно к катоду; в нем сильное электрическое поле ускоряет электроны, эмитированные катодом, и ионы, падающие на катод. Его толщина должна значительно превышать дебаевский радиус плазмы предслоя и быть меньше пробега электронов и ионов до столкновения с атомами газа.

- Предслой - переходная часть дугового столба, в которой происходит торможение плазменных электронов и предускорение ионов. Толщина этой части прикатодной области определяется длиной пробега катодного электрона до термализации за счет столкновений с молекулами (атомами) газа.

Прикатодный слой можно рассматривать как плоский диод, причем, хотя там происходит ускорение и ионов и электронов (в противоположных направлениях), объемный заряд в нем определяется ионами из-за их значительно большей массы. Поэтому, пренебрегая вкладом электронов, мы можем использовать закон 3/2 
для определения плотности ионного тока, стабильно ускоряемого в слое:

$$
j_{i}=\frac{5.4 \cdot 10^{-8}}{\sqrt{\mu_{i}}} \cdot \frac{U^{3} / 2}{d^{2}} .
$$

Здесь $j_{i}-$ плотность ионного тока, $\mathrm{A} / \mathrm{cm}^{2} ; U-$ напряжение на слое, $\mathrm{V} ; d$ - толщина слоя, $\mathrm{cm} ; \mu_{i}-$ масса иона в отношении к протонной массе.

Такая плотность ионного тока должна обеспечиваться поступлением на границу слоя со стороны плазмы предслоя потока ионов со скоростью, определяемой температурой этой слабоионизованной плазмы, которая изза высокой частоты столкновений с газовыми атомами практически одинакова для всех компонентов и равна температуре газа [3]:

$$
j_{i}=0.96 \cdot 10^{-13} \cdot n_{i} \sqrt{\frac{2 \cdot T_{p}}{\mu_{i}}}
$$

где $n_{i}-$ концентрация ионов в предслое, $\mathrm{cm}^{-3}$; $T_{p}=\frac{T_{g}}{1.16 \cdot 10^{4}}-$ температура плазмы, $\mathrm{eV} ; T_{g}-$ температура газа, $\mathrm{K} ; \mu_{i}$ - массовое число иона. Размер дебаевского радиуса оценивается по формуле

$$
\lambda_{D}=7.43 \cdot 10^{2} \cdot \sqrt{\frac{T_{p}}{n_{e}}}, \quad \mathrm{~cm}
$$

Концентрация электронов $n_{e}=n_{i}$ - из условия квазинейтральности плазмы, так как ионы при интересующих нас температурах будут однозарядными. Ионизация в разрядном столбе происходит термическим путем независимо от того, каким путем энергия в него вкладывается, поэтому плотность ионов в предслое можно оценить с помощью формулы Саха

$$
n_{i} \approx \sqrt{n_{a} \cdot 3 \cdot 10^{21} \cdot T_{g}^{1.5} \cdot \exp \left(-\frac{11600 \cdot \Phi_{N}}{T_{g}}\right)}, \mathrm{cm}^{-3},
$$

где $\Phi_{N}=14.5 \mathrm{eV}$ - энергия ионизации азота, $n_{a}-$ концентрация атомов азота. Дополним набор формул (1) - (4) выражением для оценки длины свободного пробега для атомов (ионов) азота

$$
\lambda_{\text {free }}=\frac{1}{\sqrt{2} \cdot n_{a} \cdot \pi \cdot a_{N}^{2} \cdot\left(1+\frac{C_{N}}{T_{g}}\right)}, \mathrm{cm},
$$

где $a_{N}$ - радиус атома азота, $C_{N} \approx 120-$ поправочная постоянная для азота. После чего, определив плотность потока рабочего газа в канале плазмотрона

$$
q_{g}=\frac{Q_{g}}{S_{c}}, \quad \mathrm{~g} /\left(\mathrm{s} \cdot \mathrm{cm}^{2}\right)
$$

где $Q_{g}$ - расход рабочего газа в единицу времени, $S_{c}-$ площадь сечения канала, и тепловую скорость газа в канале

$$
v_{g}=9.28 \cdot 10^{3} \cdot \sqrt{\frac{T_{g}}{\mu_{N}}}, \quad \mathrm{~cm} / \mathrm{s},
$$

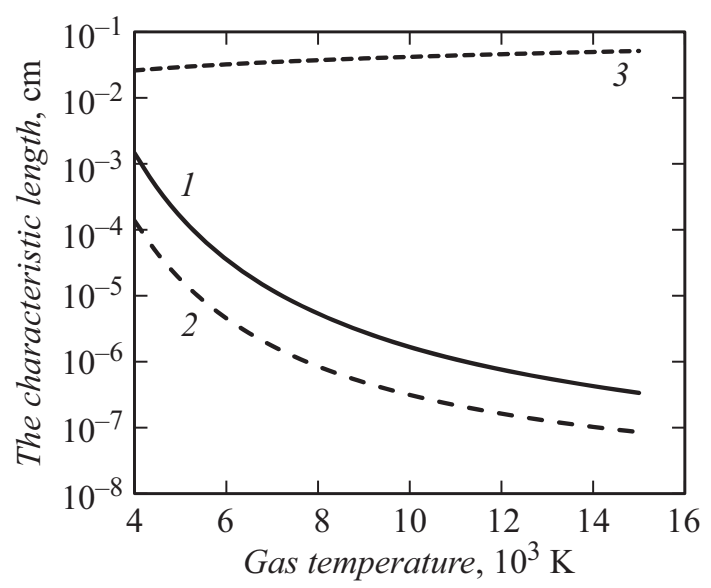

Зависимость характерных размеров: 1 - ширина слоя в соответствии с ,законом 3/2“, 2 - радиус Дебая, 3 - длина пробега иона в газе от температуры газа в предслое. Оценка проведена для удельного расхода воздуха (азота) $q_{g} \approx 1 \mathrm{~g} /\left(\mathrm{cm}^{2} \mathrm{~s}\right)$ в плазмотроне ЭДП-200 [2].

оценим пролетную плотность атомов газа

$$
n_{a}=\frac{q_{g}}{v_{g} \cdot m_{N}}, \quad \mathrm{~cm}^{-3}
$$

где $m_{N}$ - масса атома азота, g.

Затем, приняв напряжение на прикатодном слое равным $10 \mathrm{~V}$ и варьируя температуру газа, оценим с помощью выражений (1)-(5) соотношение между тремя интересующими нас характерными размерами. Результат представлен на рисунке, из которого видно, что требуемое соотношение между шириной прикатодного слоя, дебаевским радиусом и длиной свободного пробега иона до столкновения с атомом газа выполнено в интересующем нас диапазоне температур газа в предслое.

Далее считаем, что резкое увеличение испарения материала катодной вставки наступает при достижении ее поверхностью температуры плавления нитридной корки: $T_{c}=3060 \mathrm{~K}$.

Плотность тока эмиссии электронов при этой температуре с учетом эффекта Шоттки выражается следующей формулой [2]:

$$
j_{e c}=A \cdot T_{c}^{2} \cdot \exp \left[-\frac{\Phi_{e x}-e^{1.5} \cdot \sqrt{E_{c} / 300 \cdot 6.24 \cdot 10^{11}}}{T_{c} / 11600}\right],
$$

$\mathrm{A}\left(\mathrm{A} / \mathrm{K}^{2}\right)=129.4, \Phi_{e x}(\mathrm{eV})$ - работа выхода электронов из катода, для гафния: $\Phi_{e x}=3.53 \mathrm{eV}, e-$ заряд электрона (ед. СГСЭ), $E_{c}=U / d-$ напряженность электрического поля у катода $(\mathrm{V} / \mathrm{cm})$.

Дополнив формулы (1), (2), (4), (6)-(9) выражением для тока разряда $I_{d}=\left(j_{e c}+j_{i}\right) \cdot S_{c}$, где $S_{c}=\pi \cdot d^{2} / 4$ - площадь катодной вставки, и соотношением $\eta=j_{i} /\left(j_{e c}+j_{i}\right)$ - доля ионного тока в токе разряда, и используя в качестве варьируемых параметров $U$, $T_{g}$ и $q_{g}$, получим систему, связывающую предельный ток разряда с диаметром вставки и значениями параметров, 
Таблица 2.

\begin{tabular}{c|c|c|c|c}
\hline Диаметр вставки, $\mathrm{d}, \mathrm{cm}$ & 0.16 & 0.2 & 0.25 & 0.3 \\
\hline Предельный ток дуги, $I_{c r}, \mathrm{~A}$ & 80.85 & 198.50 & 306.32 & 402.88 \\
\hline $\begin{array}{c}\text { Катодное падение } \\
\text { потенциала, } \mathrm{U}_{\mathrm{K}}, \mathrm{B}\end{array}$ & 25 & 7.6 & 6.7 & 5.61 \\
\hline $\begin{array}{c}\text { Толщина катодного слоя, } \\
\lambda_{\mathrm{K}}, 10^{-5} \mathrm{~cm}\end{array}$ & 8.667 & 1.462 & 1.109 & 1.051 \\
\hline $\begin{array}{c}\text { Концентрация атомов газа, } \\
10^{16} \text { сm }^{-3}\end{array}$ & 9.611 & 13.14 & 13.14 & 13.25 \\
\hline Температура газа, К & 10300 & 12400 & 12400 & 12200 \\
\hline Доля ионнго тока & 0.06 & 0.224 & 0.227 & 0.219
\end{tabular}

сопутствующих анонсированной критической эффективной температуре эмиссионной поверхности катодной вставки. Результаты решения этой системы представлены в табл. 2.

\section{Обсуждение результатов и выводы}

Сравнение значений диаметра вставки и соответствующих ему расчетных значений критического тока с соответствующими данными табл. 1 дает основание считать, что принятая для расчета модель достаточно хорошо описывает наблюдаемое экспериментально явление резкой потери ресурсной способности вставки. Результаты косвенно подтверждают объяснение понижения скорости испарения материала гафниевой вставки в азотнокислородной среде образованием нитридной/оксидной корки на поверхности вставки.

\section{Список литературы}

[1] Аньшаков А.С., Бутова М.Н. и др. // Тр. V Всесоюз. конф. по генераторам низкотемпературной плазмы. Новосибирск: Институт теплофизики СО АН СССР, 1972. Т. 2. С. 48-52.

[2] Гнеденко В.Г., Иванов А.А., Переславцев А.В., Тресвятский C.C. // Вопросы атомной науки и техники. Серия: Плазменная электроника и новые методы ускорения. 2006. № 5. C. 75-79. 